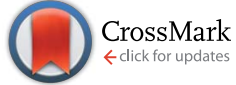

Cite this: RSC Adv., 2014, 4, 37411

Received 30th May 2014

Accepted 5th August 2014

DOI: $10.1039 / c 4 r a 05124 f$

www.rsc.org/advances

\section{Electronic and optical properties of chair-like and boat-like graphane}

\begin{abstract}
A. H. Reshak ${ }^{\star a b}$ and S. Auluck ${ }^{c}$
We have studied two favorable conformations of graphane, the chair-like graphane and boat-like graphane. In the chair-like, the $\mathrm{H}$ atoms attached to the $\mathrm{C}$ atoms alternate on both sides of the sheet while in the boatlike, the $\mathrm{C}$ bonded $\mathrm{H}$ atoms alternate in pair. Both conformations of graphane have a 2D puckered honeycomb like structure with one hydrogen atom bonded covalently $\left(\mathrm{sp}^{3}\right)$ to each carbon atom. The chair-like belongs to the $P \overline{3} m 1$ (164) space group and the boat-like belongs to the Pmmn (59) space group. We have used the state-of-the-art full potential linear augmented plane wave (FPLAPW) method with different possible approximations for the exchange-correlation (XC) potential. The XC potential was described by the local density approximation (LDA) of Ceperley-Alder (CA), the generalized gradient approximation (GGA) of Perdew-Becke-Ernzerhof (PBE) and the Engel-Vosko generalized gradient approximation (EVGGA). The calculated partial density of states for both configurations, show that there exists a strong hybridization between $\mathrm{C}$ and $\mathrm{H}$ orbitals which confirms the existence of the covalent bonds. The electronic charge density distribution of both configurations (chair-like and boat-like) has been calculated, the charge accumulates along $\mathrm{C}-\mathrm{C}$ and $\mathrm{C}-\mathrm{H}$ bonds. According to the electronegativity values of $\mathrm{C}(2.55)$ and $\mathrm{H}(2.1)$, it is clear that there is strong covalent bonding between $\mathrm{C}$ and $\mathrm{H}$ atoms. The linear optical properties give a deep insight into the electronic structure. The calculated values of the energy gap and the bond lengths show good agreement with previous results.
\end{abstract}

\section{Introduction}

Recently a new two dimensional honeycomb lattice material of a monolayer of carbon atoms was discovered. It was called graphene. ${ }^{\mathbf{1 , 2}}$ Graphene is a hexagonal lattice consisting of a single atomic layer of $\mathrm{sp}^{2}$ hybridized carbon atoms. Around each carbon atom, three strong $\sigma$ bonds are established with the other three surrounding carbon atoms. ${ }^{3}$ This two dimensional lattice of monolayer carbon atoms opened a new era in nanotechnology. The outstanding mechanical, ${ }^{4}$ electrical ${ }^{5}$ and physical properties ${ }^{6,7}$ of graphene warrants its use in a variety of areas such as hydrogen technology, ${ }^{8}$ electronics, ${ }^{5}$ sensing ${ }^{9}$ and drug delivery, ${ }^{\mathbf{1 0 1 1}}$ among many others. After this discovery several researches focused on modifying graphene for further applications in nanoelectronics. Researchers modified several graphene based materials among them the hydrogenated graphene (called graphane). ${ }^{\mathbf{1 2 - 1 9}}$ In 2003, Sluiter and Kawazoe ${ }^{\mathbf{1 2}}$ suggested the existence graphane and in 2009, Elias et al., ${ }^{\mathbf{1 4}}$ synthesized graphane by exposure the graphene sheet to the

${ }^{a}$ New Technologies - Research Centre, University of West Bohemia, Univerzitni 8, 306 14 Pilsen, Czech Republic.E-mail: maalidph@yahoo.co.uk

${ }^{b}$ Center of Excellence Geopolymer and Green Technology, School of Material Engineering, University Malaysia Perlis, 01007 Kangar, Perlis, Malaysia

${ }^{c}$ Council of Scientific and Industrial Research - National Physical Laboratory Dr K S Krishnan Marg, New Delhi 110012, India hydrogen plasma. Since then, the researchers proposed several new configurations for the newly discovered graphane.

Leenaerts et $a .^{20}$ investigated the different stoichiometric configurations of graphane and graphene fluoride within density functional theory (DFT). They found large differences between graphane and graphene fluoride which are caused by the presence of charges on the fluorine atoms. Authors also perform GW calculations for the electronic band gap of both graphene derivatives. These band gaps and also the calculated Young's moduli are at variance with available experimental data. Duminda et $a{ }^{21}{ }^{21}$ have studied the stability of chair, boat, and twist-boat graphane structures using first-principles calculations. Their results indicate that locally stable twist-boat membranes significantly contribute to the experimentally observed lattice contraction. They found that the band gaps of graphane nanoribbons decrease monotonically with the increase of the ribbon width and are insensitive to the edge structure.

Shkrebtii et al. ${ }^{22}$ proved the possibility of electron gap tuning of graphene-based materials, using extensive first principles modeling of the structural, electronic and optical properties of partially hydrogenated graphene. $\mathrm{Zhu}$ and $\mathrm{Li}^{23}$ reported that the malleable nature of atomically thin graphene makes it a potential candidate material for nanoscale origami, a promising bottom-up nanomanufacturing approach to fabricating nanobuilding blocks of desirable shapes. Klimov et al. ${ }^{24}$ have 
determined the electromechanical properties of a suspended graphene layer by scanning tunneling microscopy (STM) and scanning tunneling spectroscopy (STS) measurements, as well as computational simulations of the graphene-membrane mechanics and morphology. Lee et al. ${ }^{25}$ measured the elastic properties and intrinsic breaking strength of free-standing monolayer graphene membranes by nanoindentation in an atomic force microscope. The force-displacement behavior is interpreted within a framework of nonlinear elastic stressstrain response, and yields second- and third-order elastic stiffnesses of 340 newtons per meter $\left(\mathrm{N} \mathrm{m}^{-1}\right)$ and $-690 \mathrm{~N} \mathrm{~m}^{-1}$, respectively.

Recently, a fully hydrogenated graphene sheet, called graphane, which is an 'extended two dimensional covalently bonded hydrocarbon', having a formula unit $\mathrm{CH}$, was predicted by Sofo et al. ${ }^{13}$ Sluiter et al., ${ }^{12}$ and Sofo et al., ${ }^{13}$ suggest that among the many configurations (stirrup, boat-1, boat-2, twistedboat, chair) graphane is most stable in the chair-like configuration. Graphane could have potential applications as a hydrogen storage material and in two dimensional electronics. Chaoyu He et al. ${ }^{26}$ reported that the hexagonal hydrocarbon rings in the most stable of the five graphane allotropes are equivalent. Therefore in this study we will focus on the comparison between the most stable configuration (chair) and one of the not so stable configurations. For the latter we have selected the boat-1 configuration.

We think that it is timely to perform first-principles calculations on these two configurations to investigate the electronic structure, electronic charge density distribution, chemical bonding and the optical properties, using the state of-the-art full-potential augmented plane wave plus local orbitals approach (FP-LAPW + lo) based on the density functional theory (DFT). As first-principles' method is a useful tool for predicting physical properties of materials, it has been widely used in the calculation of physical properties of material. ${ }^{27-32}$ We should emphasize that we did not aware of any theoretical or experiment work on the optical properties of these materials. This motivated us to calculate the optical properties of chair-like and boat-like graphane. Future experimental work will testify our calculated results. The optical properties can provide detailed information about the electronic structure of the materials. The optical properties of solids are a major topic, both in basic research as well as for industrial applications. While for the former the origin and nature of different excitation processes is of fundamental interest, the latter can make use of them in many optoelectronic devices.

\section{Details of calculations}

We selected to study two conformations of graphane, the chairlike graphane and boat-like graphane as illustrated in Fig. 1. In the chair-like graphane, the $\mathrm{H}$ atoms attached to the $\mathrm{C}$ atoms alternates on both sides of the sheet (Fig. 1a) while in the boatlike graphane, the $\mathrm{C}$ bonded $\mathrm{H}$ atoms alternates in pair (Fig. 1b). Chair-like and boat-like have 2D puckered honeycomb like structure with one hydrogen atom bonded covalently $\left(\mathrm{sp}^{3}\right)$ to each carbon atom of the sheet. The chair-like conformer has

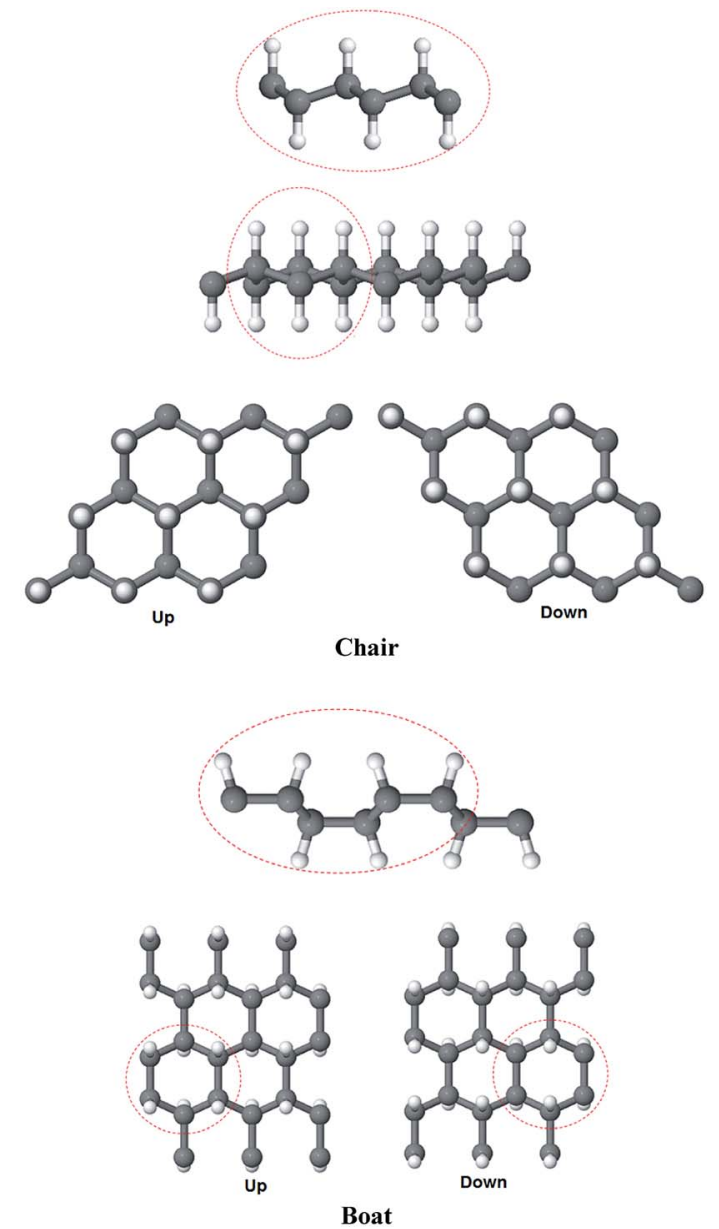

Fig. 1 The structure of chair-like/boat-like graphane. A dashed circle is used to label; in the chair-like, the $\mathrm{H}$ atoms attached to the $\mathrm{C}$ atoms alternates on both sides of the sheet while in the boat-like, the $\mathrm{C}$ bonded $\mathrm{H}$ atoms alternates in pair.

$P \overline{3} m 1$ (164) space group with unit cell $a=2.516 \AA, b=2.516 \AA$ and $c=4.978 \AA$. Whereas the boat-like conformer has Pmmn (59) space group with unit cell $a=4.272 \AA, b=2.505 \AA$ and $c=$ $4.976 \AA$. In this calculation we have used the state-of-the-art full potential linear augmented plane wave (FPLAPW) method in a scalar relativistic version as embodied in the WIEN2k code. ${ }^{33}$ This is an implementation of the density functional theory (DFT) with different possible approximations for the exchangecorrelation (XC) potential. The XC potential was described by the local density approximation (LDA) of Ceperley-Alder (CA ${ }^{34}$ and the generalized gradient approximation (GGA) of PerdewBecke-Ernzerhof (PBE), ${ }^{35}$ which is based on exchange-correlation energy optimization to calculate the total energy. In addition, we have used Engel-Vosko generalized gradient approximation (EVGGA) ${ }^{36}$ which optimizes the corresponding potential for electronic band structure calculations. The KohnSham equations are solved using a basis of linear APW's. The potential and charge density in the muffin-tin (MT) spheres are expanded in spherical harmonics with $l_{\max }=8$ and nonspherical components up to $l_{\max }=6$. In the interstitial region the potential and the charge density are represented by Fourier 
series. The structure is fully relaxed until the forces on the atoms reach values less than $1 \mathrm{mRy}$ per a.u. Once the forces are minimized in this construction one can then find the selfconsistent density at these positions by turning off the relaxations and driving the system to self-consistency. From the obtained relaxed geometry the electronic structure and the chemical bonding can be determined and various spectroscopic features can be simulated and compared with experimental data. Self-consistency is obtained using $5000 \vec{k}$ points in the irreducible Brillouin zone (IBZ). We have calculated the linear optical susceptibilities using $10000 \vec{k}$ points in the IBZ. The FPLAPW method has proven to be one of the most accurate methods for calculating the electronic structure of solids within DFT. $^{37,38}$

\section{Results and discussion}

3.1. Electronic band structure, density of states and electronic charge density

The electronic band structure along the high symmetry directions for the chair-like graphane and boat-like graphane, calculated using three types of exchange correlations mentioned above, are shown in Fig. 2. In all cases and for both configurations the conduction band minimum (CBM) and the valence band maximum (VBM) are located at $\Gamma$ point the center of the BZ, resulting in a direct band gap. The values of the energy gap for both configurations using different exchange and correlation potentials in comparison to the previous theoretical work are listed in Table 1. Following Table 1, one can see that the values of the energy gap increases when we move from LDA to GGA then to EVGGA. To the best of our knowledge, there are no experimental data for the energy band gaps available in literature to make a meaningful comparison. One can see that the values of energy gap obtained by EVGGA show good

Table 1 The calculated energy gap in comparison to previous calculations

\begin{tabular}{lll}
\hline & Chair-like & Boat-like \\
\hline LDA & 2.69 & 3.02 \\
GGA & $3.02,3.49^{a}, 3.5^{b}$ & $3.37,3.37^{a}, 3.7^{b}$ \\
EVGGA & 3.60 & 3.90
\end{tabular}

${ }^{a}$ Ref.20. ${ }^{b}$ Ref. 13.
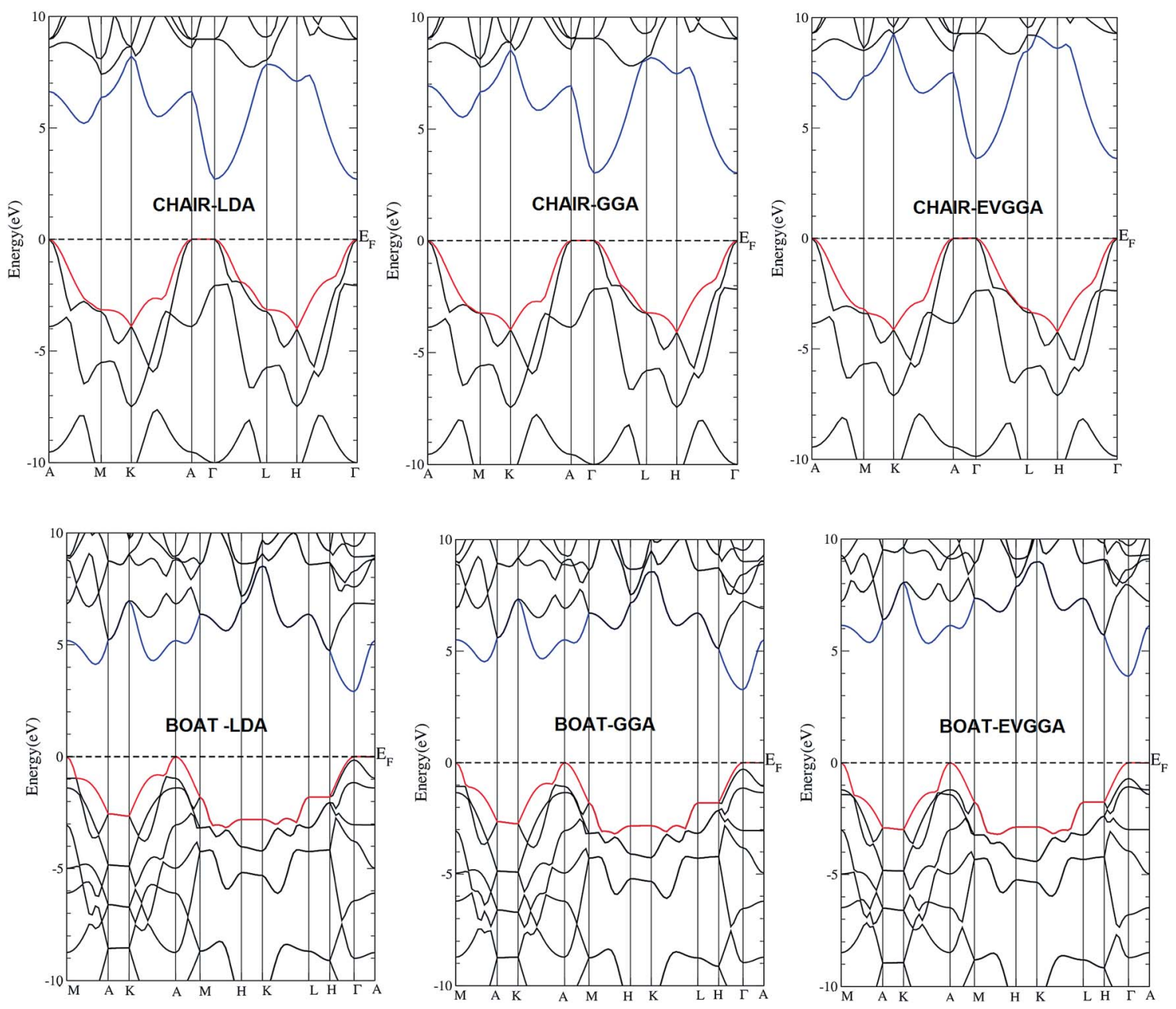

Fig. 2 Calculated band structure of chair-like/boat-like graphane using LDA, GGA and EVGGA. 
agreement with previous theoretical results. ${ }^{\mathbf{1 3 , 2 6}}$ This is mainly due to the fact that LDA and GGA have simple forms that are not sufficiently flexible to accurately reproduce both the exchangecorrelation energy and its charge derivative. Engel and Vosko considered this shortcoming and constructed a new functional form of GGA that is able to better reproduce the exchange potential at the expense of less agreement in the exchange energy. Thus, EVGGA, yields better band splitting and some other properties that mainly depend on the accuracy of the exchange-correlation potential.

Due to different configurations the boat-like graphane exhibits extra bands in the CB and VB in comparison to chairlike graphane. The total density of states (TDOS) along with the $\mathrm{C}-\mathrm{s} / \mathrm{p}$ and H-s partial density of states (PDOS) of both configurations are shown in Fig. 3a-f. Fig. $3 \mathrm{a}$ and $\mathrm{b}$ shows the TDOS of chair-like graphane and boat-like graphane using three kinds of exchange correlations. We find that moving from LDA to GGA to EVGGA confirms our previous finding regarding that increase in the band gaps. As the gap obtained with EVGGA agrees best with other workers, ${ }^{13,26}$ we decided to show the results obtained by
EVGGA only. Fig. 3c shows the TDOS of both configurations using EVGGA. We find that the calculated TDOS shows the general structure of both configurations. However the boat-like graphane exhibits more structure with higher amplitudes in comparison to chair-like graphane, which confirm our previous finding that boat-like has more bands than chair-like.

Fig. $3 \mathrm{~d}$ and e, illustrates the C-s/p and H-s partial density of states for both configurations. We notice that for chair-like there exists a strong hybridization between C-s and C-p states in the energy range between $-\mathbf{1 1 . 0}$ and $-7.0 \mathrm{eV}$ and around $7.5 \mathrm{eV}$. In the energy range extended from -9.0 till $-7.0 \mathrm{eV}$ and also around $6.5 \mathrm{eV}$ the $\mathrm{C}$-s/p states strongly hybridized with $\mathrm{H}$-s state. The C-s state hybridized with $\mathrm{H}$-s state between 4.0 and $6.0 \mathrm{eV}$, 7.0 and $8.5 \mathrm{eV}, 10.5$ and $11.5 \mathrm{eV}$, and around $15.0 \mathrm{eV}$. While for boat-like, C-s/p states hybridized with $\mathrm{H}$-s state around $\mathbf{- 1 1 . 0}$ $\mathrm{eV}, \mathrm{C}-\mathrm{p}$ state hybridized with $\mathrm{H}$-s states around $-4.5 \mathrm{eV}$, whereas C-s state hybridized with $\mathrm{H}$-s state between 4.0-8.0 eV, 8.5-9.5 $\mathrm{eV}$ and 14.0-15.0 eV. In the chair-like graphane the strong hybridization between $\mathrm{C}-\mathrm{s} / \mathrm{p}$ and $\mathrm{H}$-s states at the VB and the $\mathrm{CB}$ resulting in very strong covalent bonds, the may be one reason
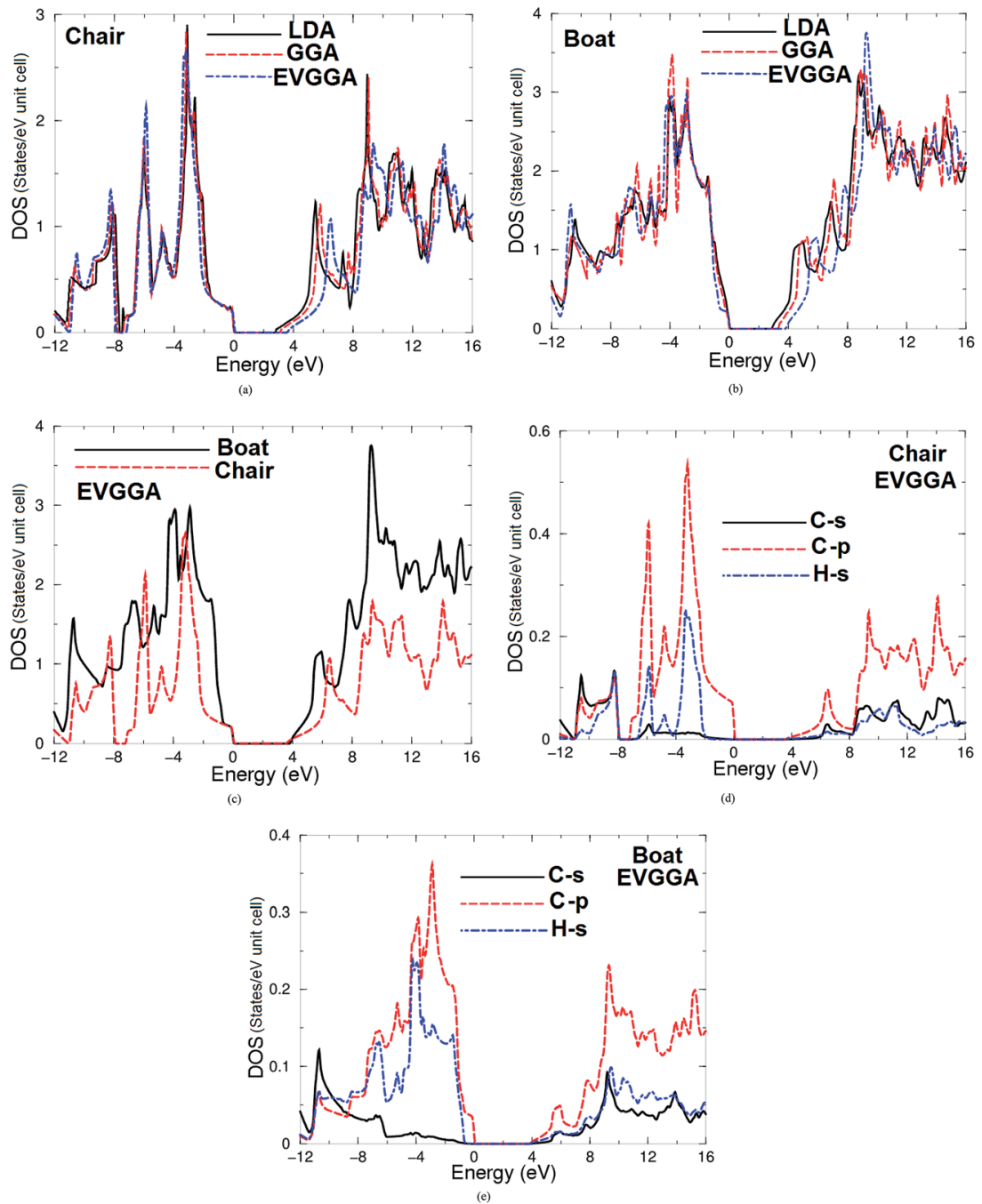

Fig. 3 Calculated total and partial density of states for chair-like/boat-like graphane using LDA, GGA and EVGGA. 
for the high stability of this configuration, while boat-like exhibit strong hybridization between C-s/p and $\mathrm{H}$-s only around $-11.0 \mathrm{eV}$ that means the covalent bonds is concentrated only in this energy region. For both configurations it is clear that the VBM and the CBM is mainly formed by C-p states.

From the PDOS-VB one can elucidate the origin of chemical bonding following the same method we used in our previous work. ${ }^{39}$ One should emphasize that for the chair-like the PDOSVB located between $-8.0 \mathrm{eV}$ and $E_{\mathrm{F}}$ is larger compared to C-p state $(0.55$ electron per $\mathrm{eV}), \mathrm{H}$-s state $(0.22$ electron per $\mathrm{eV})$ and C-s states (0.02 electron per eV), while for boat-like it is for C-p state $(0.37$ electron per $\mathrm{eV}), \mathrm{H}$-s state $(0.24$ electron per $\mathrm{eV})$ and C-s states ( 0.04 electron per $\mathrm{eV})$. Therefore some electrons from $\mathrm{C}$ and $\mathrm{H}$ atoms are transferred into valence bands and contribute in covalence interactions. The interaction due to the strong hybridization and the covalent bonds is defined by the degree of hybridization. Hence, there is a strong covalent bonding between the atoms which exhibit strong hybridization. The PDOS helps to analyze the nature of the bonds according to a classical chemical concept. This concept is very useful to classify compounds into different categories with respect to different chemical and physical properties. To support this statement we have taken a more careful look at the bonding situation since the existence of real hybridization between states of atoms should lead to covalent bond's origin between these atoms.

The electronic charge density distribution of both configurations (chair-like and boat-like) has been calculated in the (11 $0)$ and $\left(\begin{array}{lll}-1 & 0 & 1\end{array}\right)$ crystallographic plane, respectively as shown in Fig. $4 \mathrm{a}$ and $\mathrm{b}$. One can see that the charge accumulates around $\mathrm{C}-\mathrm{C}$ and $\mathrm{C}-\mathrm{H}$ along the bonds. According to the electro-negativity values of $\mathrm{C}(2.55)$ and $\mathrm{H}$ (2.1), it is clear that there is a strong covalent bonding between $\mathrm{C}$ and $\mathrm{H}$ atoms. This can be seen easily by color charge density scale where blue color $(+1.00)$ corresponds to the maximum charge accumulation site. Also one can see that there is a strong covalent bonding between the $\mathrm{C}-\mathrm{C}$ and $\mathrm{C}-\mathrm{H}$ atoms. We should emphasize that in the centre of the honeycomb the charge is zero as can be seen by red color $(+0.00)$ corresponds to the minimum charge accumulation site. The bond lengths and angles are listed in Table 2. Following this table one can see that the chair-like graphane has only one type of $\mathrm{C}-\mathrm{C}$ bond, while boat-like have two types of $\mathrm{C}-\mathrm{C}$ bonds. The bond length of $\mathrm{C}-\mathrm{C}$ which are attached to $\mathrm{H}$ on the same side is longer (due to $\mathrm{H}-\mathrm{H}$ repulsion) than that which $\mathrm{H}$ attached in opposite sides.

\subsection{Optical properties}

The optical properties can provide detailed information about the electronic structure of the materials. The optical properties of solids are a major topic, both in basic research as well as for industrial applications. While for the former the origin and nature of different excitation processes is of fundamental interest, the latter can make use of them in many optoelectronic devices. The chair-like conformer is belong to $P \overline{3} m 1$ (164) space group, this symmetry allows two non-zero components of the complex second-order dielectric (optical) tensor corresponding

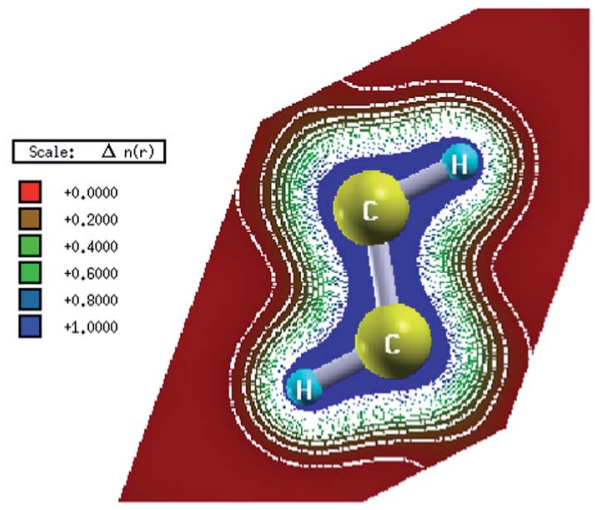

(a) chair-like

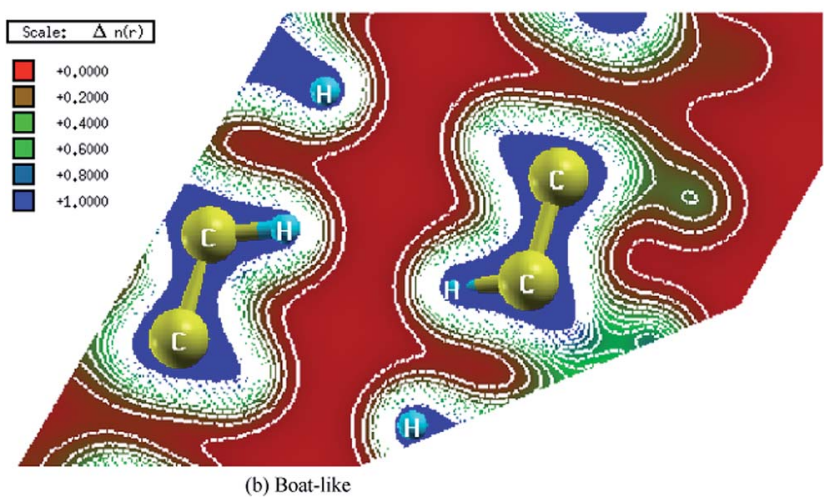

Fig. 4 Calculated electronic charge density distribution for chair-like graphane in the $(110)$ crystallographic plane and boat-like graphane in the $\left(\begin{array}{llll}-1 & 0 & 1\end{array}\right)$ crystallographic plane.

Table 2 Calculated bond lengths and angles in comparison with the previous calculations

\begin{tabular}{|c|c|c|}
\hline & Chair-like & Boat-like \\
\hline \multicolumn{3}{|c|}{ Bond lengths (@) } \\
\hline $\mathrm{C}-\mathrm{C}$ & $(\stackrel{\mathrm{H}}{\mathrm{C}}-\underset{\mathrm{H}}{\mathrm{C}}): 1.526^{*}, 1.52^{a}$ & $\begin{array}{l}(\mathrm{C}-\mathrm{C}): 1.522^{*}, \\
1.52^{a}(\stackrel{\mathrm{H}}{\mathrm{C}}-\stackrel{\mathrm{H}}{\mathrm{C}}): 1.554^{*}, 1.56^{a}\end{array}$ \\
\hline $\mathrm{C}-\mathrm{H}$ & $1.135^{*}, 1.11^{a}$ & $1.113^{*}, 1.10^{a}$ \\
\hline \multicolumn{3}{|c|}{ Bond angles $\left({ }^{\circ}\right)$} \\
\hline $\mathrm{H}-\mathrm{C}-\mathrm{C}$ & 107.81 & 106.96 \\
\hline
\end{tabular}

to the electric field $\vec{E}$ being directed along a and c-crystallographic axes, these are $\varepsilon^{x x}(\omega)$ and $\varepsilon^{z z}(\omega)$. While for boat-like conformer (Pmmn (59)) the symmetry allows three non-zero components of the complex second-order dielectric tensor corresponding to the electric field $\vec{E}$ being directed along a, b, and c-crystallographic axes, these are $\varepsilon^{x x}(\omega), \varepsilon^{y y}(\omega)$ and $\varepsilon^{z z}(\omega)$. We identify these with the $x, y$ and $z$ Cartesian directions. The imaginary part of the two/three complex tensor components completely defines the linear optical properties. The imaginary part of the optical function's dispersion originates from interband transitions between the valence and conduction bands. 
According to the dipolar selection rule only transitions changing the angular momentum quantum number $l$ by unity are allowed. The imaginary parts of the optical function's dispersion were calculated using the following expression ${ }^{40}$
In Fig. 5b, we show the three tensor components of boat-like graphane $\varepsilon_{2}^{x x}(\omega), \varepsilon_{2}^{y y}(\omega)$ and $\varepsilon_{2}^{z z}(\omega)$. The threshold of the optical absorption for the three components $\varepsilon_{2}^{x x}(\omega), \varepsilon_{2}^{y y}(\omega)$ and $\varepsilon_{2}^{z z}(\omega)$ are located at $3.2 \mathrm{eV} . \varepsilon_{2}^{x x}(\omega)$ and $\varepsilon_{2}^{y y}(\omega)$ displays one principal peak

$$
\varepsilon_{2}^{i j}(\omega)=\frac{8 \pi^{2} \hbar^{2} e^{2}}{m^{2} V} \sum_{k} \sum_{c v}\left(f_{\mathrm{c}}-f_{\mathrm{v}}\right) \frac{p_{c v}{ }^{i}(k) p_{c v}{ }^{j}(k)}{E_{v c}{ }^{2}} \delta\left[E_{\mathrm{c}}(k)-E_{\mathrm{v}}(k)-\hbar \omega\right]
$$

where $m, e$ and $\hbar$ are the electron mass, charge and Planck's constant, respectively. $f_{\mathrm{c}}$ and $f_{\mathrm{v}}$ represent the Fermi distributions of the conduction and valence bands, respectively. The term $p_{c v}{ }^{i}(k)$ denotes the momentum matrix element transition from the energy level $c$ of the conduction band to the level $v$ of the valence band at certain k-point in the $\mathrm{BZ}$ and $V$ is the unit cell volume.

The two components of the imaginary part of the dielectric functions $\varepsilon_{2}^{x x}(\omega)$ and $\varepsilon_{2}^{z z}(\omega)$ for chair-like graphane are shown in Fig. 5a. The edges of the optical absorption (threshold) for these two components are situated at $3.60 \mathrm{eV}$. Two fundamental peaks were located at around $11.0 \mathrm{eV}$ for $\varepsilon_{2}^{x x}(\omega)$ and $13.0 \mathrm{eV}$ for $\varepsilon_{2}^{z z}(\omega)$. One can see that the fundamental peak of $\varepsilon_{2}^{x x}(\omega)$ is situated between two humps, while that of $\varepsilon_{2}^{z z}(\omega)$ are located between the small peak (around 8.0 $\mathrm{eV}$ ) from the left hand side and several humps on the right hand side. There exists a considerable anisotropy between $\varepsilon_{2}^{x x}(\omega)$ and $\varepsilon_{2}^{z z}(\omega)$. situated around 11.0 and $12.0 \mathrm{eV}$, while $\varepsilon_{2}^{z z}(\omega)$ displays two principal peaks located around 8.0 and $16.0 \mathrm{eV}$. The first peak consists of one small hump while the second peak is situated between two humps. One can see a considerable anisotropy between the three components. Knowing the imaginary part of the dielectric function's dispersions $\varepsilon_{2}^{x x}(\omega), \varepsilon_{2}^{y y}(\omega)$ and $\varepsilon_{2}^{z z}(\omega)$ the real part $\varepsilon_{1}^{x x}(\omega), \varepsilon_{1}^{y y}(\omega)$ and $\varepsilon_{1}^{z z}(\omega)$ can be obtained following Kramers-Kronig relations. These are shown in Fig. $5 \mathrm{c}$ and d. Again it shows a considerable anisotropy. The calculated $\varepsilon_{1}^{x x}(0)$, $\varepsilon_{1}^{y y}(0)$ and $\varepsilon_{1}^{z z}(0)$ are presented in Table 3.

Table 3 Calculated $\varepsilon_{1}^{x x}(0), \varepsilon_{1}^{y y}(0)$ and $\varepsilon_{1}^{z z}(0)$

\begin{tabular}{lll}
\hline Component & Chair-like & Boat-like \\
\hline$\varepsilon_{1}^{x x}(0)$ & 3.1 & 3.08 \\
$\varepsilon_{1}^{y y}(0)$ & - & 3.23 \\
$\varepsilon_{1}^{z z}(0)$ & 3.3 & 3.27
\end{tabular}
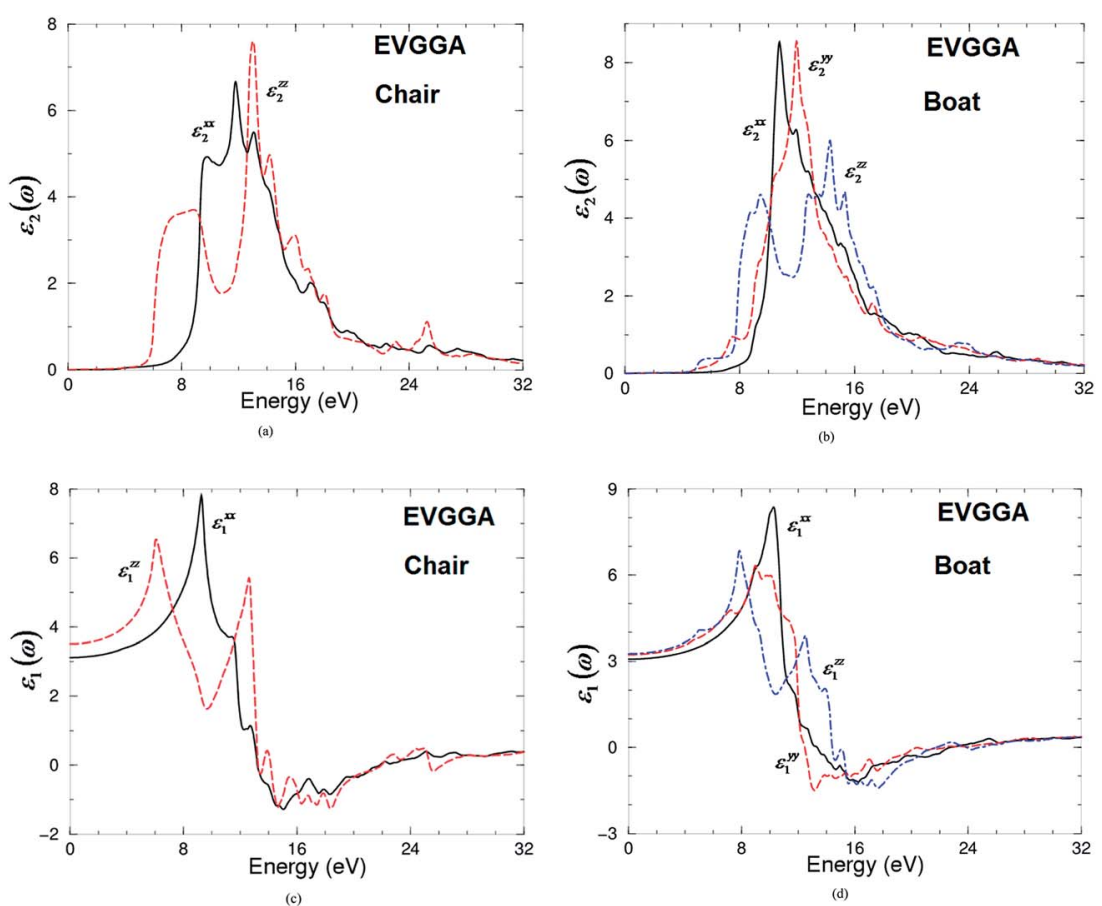

Fig. 5 Calculated imaginary and real parts of the optical dielectric function for chair-like/boat-like graphane using EVGGA. 


\section{Conclusions}

The state-of-the-art full potential linear augmented plane wave (FPLAPW) method as embodied in the WIEN2k code with three different possible approximations for the exchange-correlation (XC) potential were used to calculate the electronic and optical properties of two conformations of graphane, the chair-like graphane and boat-like graphane. The three XC potentials used are local density approximation (LDA) of Ceperley-Alder (CA), gradient approximation (GGA) of Perdew-Becke-Ernzerhof (PBE), and Engel-Vosko generalized gradient approximation (EVGGA). For chair-like graphane and boat-like graphane the conduction band minimum (CBM) and the valence band maximum (VBM) are located at $\Gamma$ point the center of the BZ, resulting in a direct band gap of about 2.69/3.02 eV (LDA), 3.02/ $3.37 \mathrm{eV}$ (GGA) and 3.60/3.90 eV (EVGGA) for chair-like/boat-like. Our calculated energy gap for chair-like/boat-like using EVGGA show good agreement with the previous theoretical results 3.47 $\mathrm{eV}$ and $3.5 \mathrm{eV} / 3.37 \mathrm{eV}$ and $3.7 \mathrm{eV}$. The calculated partial density of states exhibits a strong hybridization between the orbitals which shows the existence of the covalent bonds. In additional we have calculated the electronic charge density distribution of chair-like/boat-like graphane which confirms the existence of the covalent bonds. The linear optical properties are presented and discussed in details. The optical properties exhibit considerable anisotropy, from the optical properties we can get deep insight the electronic structure, therefore the calculated optical function's dispersion confirm increasing the energy gap when we move from chair-like to boat-like graphane also it confirm the complicated structure of boat-like graphane. The calculated values of the bond lengths obtained using EVGGA show good agreement with pervious results.

\section{Acknowledgements}

The result was developed within the CENTEM project, reg. no. CZ.1.05/2.1.00/03.0088, co-funded by the ERDF as part of the Ministry of Education, Youth and Sports OP RDI program. Computational resources were provided by MetaCentrum (LM2010005) and CERIT-SC (CZ.1.05/3.2.00/08.0144) infrastructures. S.A. thanks Council of Scientific and Industrial Research (CSIR) - National Physical Laboratory for financial support.

\section{References}

1 K. Nakada, M. Fujita, G. Dresselhaus and M. S. Dresselhaus, Phys. Rev. B: Condens. Matter Mater. Phys., 1996, 54, 17954.

2 S. Okada, Phys. Rev. B: Condens. Matter Mater. Phys., 2008, 77, 041408.

3 R. Lv and M. Terrones, Mater. Lett., 2012, 78, 209-218.

4 C. Gomez-Navarro, M. Burghard and K. Kern, Nano Lett., 2008, 8, 2045.

5 Y.-H. Lin, K. A. Jenkins, A. Valdes-Garcia, J. P. Small, D. B. Farmer and P. Avouris, Nano Lett., 2009, 9, 422.

6 C. L. Kane and E. J. Mele, Phys. Rev. Lett., 2005, 95, 226801.
7 A. H. Reshak, S. A. Khan and S. Auluck, J. Mater. Chem. C, 2014, 2, 2346-2352.

8 A. Ferre-Vilaplana, J. Chem. Phys., 2005, 122, 104709.

9 I. Zanella, S. B. Fagan, R. Mota and A. Fazzio, J. Phys. Chem. C, 2008, 112, 9163.

10 Z. Liu, J. T. Robinson, X. Sun and H. Dai, J. Am. Chem. Soc., 2008, 130, 10876.

11 A. de Leon and A. F. Jalbout, Chem. Phys. Lett., 2008, 457, 179.

12 M. H. F. Sluiter and Y. Kawazoe, Phys. Rev. B: Condens. Matter Mater. Phys., 2003, 68, 085410.

13 J. O. Sofo, A. S. Chaudhari and G. D. Barber, Phys. Rev. B: Condens. Matter Mater. Phys., 2007, 75, 153401.

14 D. C. Elias, R. R. Nair, T. M. G. Mohiuddin, S. V. Morozov, P. Blake, M. P. Halsall, et al., Science, 2009, 323, 610.

15 D. K. Samarakoon and X. Q. Wang, ACS Nano, 2009, 12, 4017. 16 O. Leenaerts, H. Peelaers, A. D. Hernández-Nieves, B. Partoens and F. M. Peeters, Phys. Rev. B: Condens. Matter Mater. Phys., 2010, 82, 195436.

17 A. Bhattacharya, S. Bhattacharya, C. Majumder and G. P. Das, Phys. Rev. B: Condens. Matter Mater. Phys., 2011, 83, 033404; A. Bhattacharya, S. Bhattacharya, C. Majumder and G. P. Das, J. Phys. Chem. C, 2010, 114, 10299; S. Bhattacharya, C. Majumder and G. P. Das, J. Phys. Chem. C, 2009, 113, 15783.

18 X. D. Wen, L. Hand, V. Labet, T. Yang, R. Hoffmann, N. W. Ashcroft, A. R. Oganov and O. Lyakhov, Proc. Natl. Acad. Sci. U. S. A., 2011, 108, 6833.

19 D. K. Samarakoon, Z. F. Chen, C. Nicolas and X. Q. Wang, Small, 2011, 7, 965.

20 O. Leenaerts, H. Peelaers, A. D. Hernández-Nieves, B. Partoens and F. M. Peeters, Phys. Rev. B: Condens. Matter Mater. Phys., 2010, 82, 195436.

21 D. K. Samarakoon and X.-Q. Wang, ACS Nano, 2009, 3(12), 4017-4022.

22 A. I. Shkrebtii, E. Heritage, P. McNelles, J. L. Cabellos and B. S. Mendoza, Phys. Status Solidi C, 2012, 9(6), 1378-1383.

23 S. Zhu and T. Li, ACS Nano, 2014, 8(3), 2864-2872.

24 N. N. Klimov, S. Jung, S. Zhu, T. Li, C. Alan Wright, S. D. Solares, D. B. Newell, N. B. Zhitenev and J. A. Stroscio, Science, 2012, 336, 1557.

25 C. Lee, X. Wei, J. W. Kysar and J. Hone, Science, 2008, 321, 385.

26 C. He, L. Z. Sun, C. X. Zhang, N. Jiao, K. W. Zhang and J. Zhong, arXiv:1204.6621v1 [cond-mat.mtrl-sci]; C. He, C. X. Zhang, L. Z. Sun, N. Jiao, K. W. Zhang and J. Zhong, Phys. Status Solidi RRL, 2012, 6(11), 427-429.

27 A. H. Reshak, S. Auluck and I. V. Kityk, J. Phys.: Condens. Matter, 2008, 20, 145209.

28 A. H. Reshak, S. Auluck and I. V. Kityk, J. Solid State Chem., 2008, 181, 789-795.

29 A. H. Reshak, J. Chem. Phys., 2006, 124, 104707; A. H. Reshak, Eur. Phys. J. B, 2005, 47, 503-508.

30 A. H. Reshak, I. V. Kityk and S. Auluck, J. Phys. Chem. B, 2010, 114, 16705-16712.

31 A. H. Reshak, S. Auluck, D. Stys, I. V. Kityk, H. Kamarudin, J. Berdowski and Z. Tylczynskif, J. Mater. Chem., 2011, 21, 17219. 
32 A. H. Reshak, M. Piasecki, S. Auluck, I. V. Kityk, R. Khenata, B. Andriyevsky, C. Cobet, N. Esser, A. Majchrowski, M. S'wirkowicz, R. Diduszko and W. Szyrski, J. Phys. Chem. $B, 2009,113(46), 15237$.

33 P. Blaha, K. Schwarz, G. K. H. Madsen, D. Kvasnicka and J. Luitz, WIEN2k, An augmented plane wave plus local orbitals program for calculating crystal properties, Vienna University of Technology, Austria, 2001.

34 D. M. Ceperley and B. I. Ader, Phys. Rev. Lett., 1980, 45, 566569, parametrized in J. P. Perdew and A. Zunger, Phys. Rev. B: Solid State, 1973, 8, 4822-4832.
35 J. P. Perdew, S. Burke and M. Ernzerhof, Phys. Rev. Lett., 1996, 77, 3865.

36 E. Engel and S. H. Vosko, Phys. Rev. B: Condens. Matter Mater. Phys., 1993, 47, 13164.

37 S. Gao, Comput. Phys. Commun., 2003, 153, 190.

38 K. Schwarz, J. Solid State Chem., 2003, 176, 319.

39 A. H. Reshak, D. Stys, S. Auluck and H. Kamarudin, Mater. Chem. Phys., 2011, 130, 458.

40 F. Bassani and G. P. Parravicini, Electronic States and Optical Transitions In Solids, Pergamon Press Ltd, Oxford, 1975, pp. 149-154. 